\title{
Thyroid tumors with encapsulated follicular pattern : a diagnostıc dilemma
}

\begin{abstract}
We aimed to determine the immunoreactivity for B-type Raf kinase (BRAF), Cytokeratin-19 (CK-19), Hector Battifora mesothelial-1 (HBME-1), and Galectin-3 (GAL-3) antibodies in single and combination forms to define their potential role on diagnosis in encapsulated follicular thyroid tumors creating diagnostic difficulties. One hundred thirty five thyroid tumors diagnosed with follicular adenoma (FA, $\mathrm{n}=50$ ), encapsulated minimally invasive follicular carcinoma (MIFC, $\mathrm{n}=40$ ), and encapsulated follicular variant of papillary carcinoma $(\mathrm{FVPC}, \mathrm{n}=45)$ were evaluated for immunohistochemically. For a single antibody, HBME-1 was the most sensitive antibody for the diagnosis of FVPC vs FA $(93,33 \%)$, whereas CK-19 had the lowest sensitivity among the 4 markers. HBME-1 + GAL-3 combination has the most sensitivity value $(67,5 \%)$ for diagnosing MIFC vs FA. The combination of CK19+HBME1+GAL3 and CK19+HBME1+BRAF have the highest sensitivity values $(62,5 \%)$ for diagnosing FK vs FA. The coexpression of the four antibodies showed average sensitivity and high specificity results as $60-65 \%$ in sensitivity and $80 \%$ in spesifity for MIFC vs FA; FVPC vs FA and FVPC vs MIFC. For optimum sensitivity and specificity, the combination of the four markers was suggested for the diagnosis of MIFC vs FA. In conclusion, immunohistochemical evaluation of BRAF, CK-19, HBME-1 and GAL-3 in tyhroid tumors would aid the differentiation of malignant follicular lesions from benign follicular lesion. Combination of different forms of antibodies in accordance with the lesion type to be distinguished may increase the sensitivity of diagnosis, which needs to be confirmed with further studies.
\end{abstract}

Volume 2 Issue 3 - 2017

\author{
Rabia Bozdogan Arpaci,' Tuba Kara,' Didar \\ Gursoy,' Gulhan Örekici,' Ahmet Dag,' Taner \\ Arpaci $^{3}$ \\ 'Department of Pathology, Bioistatistics, Mersin University, \\ Turkey \\ 2Department of General Surgery, Acibadem University, Turkey \\ ${ }^{3}$ Department of Radiology, Acibadem University, Turkey
}

\begin{abstract}
Correspondence: Rabia Bozdogan Arpaci, Medical School, Department of Pathology, Mersin University, 33100 , Mersin,Turkey, Tel +903243374300, Fax +903243374309, Email rabiabarpaci@gmail.com
\end{abstract}

Received: May 03, 2017 | Published: June 30, 2017

Keywords: tyhroid tumors, carcinoma, adenoma, 1mmunoreactivity

\section{Introduction}

Five percent of the thyroid nodules are malignant, and PC is the most common type of malignant thyroid tumor. The follicular lesions, on the other hand, presents a heterogeneous group of cases with varying malignancy potential. Due to difficulties in assessment of malignancy potential with certainty, thyroid lesions with follicular pattern are sometimes termed as thyroid tumors of uncertain or indeterminate malignant potential. ${ }^{1,2}$ Follicular patterned thyroid lesions are; adenomatous (hyperplastic, adenomatoid) nodules, follicular adenomas, follicular carcinomas (minimally invasive, grossly encapsulated, angioinvasive and widely invasive), follicular variant of papillary thyroid carcinomas, follicular variant of medullary carcinomas and "hybrid" tumors. Follicular adenoma is a solitary lesion in an otherwise normal gland; it is completely encapsulated and lacks any capsular and/or vascular invasion into the tumor capsule and surrounding thyroid. The term minimally invasive follicular carcinoma for tumors that show capsular invasion only. FVPC is the most common subtype of PC. ${ }^{3}$ Follicular lesions of thyroid show very similarly histopathologic patterns. Therefore, FA, MIFC, and FVPC can be easily confused with each other. Since prognosis and treatment approaches change according to type of follicular lesion, in addition to histopathological evaluation, immunohistochemical and molecular markers are suggested to be used to confirm the diagnosis. ${ }^{4}$

BRAF is a serine-threonine kinase signaling molecule, and mutations of BRAF leads to potential activation of MAP kinase/ERK pathway, which is the main pathway through which the majority of genetic alterations exert their oncogenic actions in thyroid cancer. ${ }^{5}$ Activation of MAP kinase/ERK pathway stimulates tumor formation by upregulating cell division and proliferation. ${ }^{6}$ BRAF mutation is seen in $35-67 \%$ of papillary carcinoma (PC) cases $^{7}$, thus suggested to be used in differential diagnosis of PC. However, in the FVPC, BRAF mutation was reported in only $5-20 \%$ of cases, ${ }^{8}$ and there is limited study on the role of BRAF expression in differentiating between follicular lesions of thyroid. ${ }^{1,4}$ GAL-3 is a beta-galactosylbinding lectin which is involved in regulating cell-cell and cellmatrix interactions. Galectin-3 expression has been proposed to be a reliable molecular marker of $\mathrm{FC}^{9}$ and $\mathrm{PC},{ }^{10}$ and suggested be used to differentiate benign from malignant thyroid nodules. However, some studies reported GAL-3 expression in epithelial cells of normal thyroid tissue and questionned its reliability as an immunohistochemical marker to distinguish benign from malignant thyroid follicular lesions. ${ }^{11,12}$ CK-19 is a cytoskeletal protein that was shown to be increased in PC, and suggested as a marker in differential diagnosis of benign and malignant thyroid nodules. ${ }^{13,14} \mathrm{HBME}-1$, a monoclonal antibody generated against malignant epithelial mesothelioma cells. Its expression has also been reported in PC and MIFC. ${ }^{15,16}$ Differential diagnosis of thyroid lesions with follicular pattern with molecular and immunohistochemical methods to determine the lesions with malignancy potential is crucial in follow-up and treatment planning. Evaluation of immunohistochemical expression of above proteins may aid in differential diagnosis of follicular pattern thyroid lesions and coexpression of multiple proteins may further increase specificity in differential diagnosis. In this study we aimed to determine the individual and combined immunoreactivity of BRAF, CK-19, HBME-1 and GAL-3 for the tumors by FA, FVPC and MIFC having encapsulated follicular pattern which leading to difficulties on differential diagnosis and define their potential role in differential diagnosis by evaluating sensitivity and spesifity. 


\section{Materials and methods}

The study was performed on 135 thyroid tumors which diagnosed with FA ( $\mathrm{n}=50), \operatorname{MIFC}(\mathrm{n}=40)$, and FVPC $(\mathrm{n}=45)$ in Mersin University School of Medicine, Department of Pathology between 2007 and 2014. The lesions were were defined suspicious for malignancy with ultrasonographically before operation. Masson Trichrome histochemical satining was performed to assess the integrity of the tumor capsule. Immunohistochemistery satining was performed with a standard avidin-biotin-immunoperoxidase technique on $4 \mu \mathrm{m}$ thick, formalin-fixed paraffin-embedded thyroid tissues to evaluate the expressions of BRAF (Epitomics, 1647-1, dilution 1/200), HBME1 (Thermoscientific, HBME-1, dilution, 1/50), CK-19 (Neomarkers, A53-B/A2.26, dilution 1/100) and GAL-3 (Novacastra, NCL-GAL3, dilution $1 / 40$ ).

We evaluated the cytoplasmic staining of tumor cells with BRAF, CK-19, cytoplasmic and nuclear staining with GAL-3 and membranous staining with HBME-1, according to Scognamiglio et al.. ${ }^{17}$ The staining intensity for each antibody was scored as follows: 0 , negative; $1+$, weak; $2+$, moderate; and $3+$, strong. Positive immunostaining was defined as $2+$ to $3+$ staining intensity in more than $10 \%$ of the cells. The relations between the expressions and staining for BRAF, HBME-1, CK-19, or GAL-3 in lesion groups were evaluated by four pathologists with the microscope of Olympus Bx51.

\section{Statistical analysis}

Study data were summarized using descriptive statistics, such as frequency and percentage. The relations between lesion types and study parameters, between BRAF expression and staining percentages for HBME-1, CK-19, or GAL-3 were analyzed with chi-square test For sensitivity and spasifity calculations MedCalc program was used. All statistical analyses were per-formed using Statistical Package for Social Sciences software (version 11.5, SPSS Inc., Chicago, Illinois, USA). Statistical level of significance was set to $p<0.05$.

\section{Results}

\section{Histochemical staining with masson trichrome}

The encapsulation of different thickness were observed with green color for Masson Trichrome histochemical staining in all tumor groups (Figures 1-3).

\section{BRAF, CK-19, HBME-I, GAL-3 expresssions in FVPC vs FA}

Immunohistochemical analysis revealed that BRAF expression was positive in 14 subjects $(14 / 50,28 \%)$ in FA, and 32 subjects (71.1\%) in FVPC groups (Table 1). The staining characteristic was mostly cytoplasmic for BRAF in FVPC group. Normal thyroid tissue was not stained with BRAF. BRAF expression was higher in FVPC cases than FA and there was a significant relation between FVPC and BRAF expresssion ( $<<0.001$, Table 1$)$. Most of the subjects with BRAF positivity was from FVPC group. The sensitivity and spesifity values were $71.11 \%$ and $100 \%$ between the lesions for BRAF. In FVPC, 35 cases $(77.8 \%)$ showed positive staining for CK-19. The staining pattern was cytoplasmic and nearly diffuse in this group. A few cells showed weak and patchy staining in normal thyroid. However only 4 of 50 (8\%) cases showed positive staining for CK-19 in FA groups. A patchy and weak staining were noticed in FA. These two groups (FVPC vs FA) were compared in the respect of CK-19.
The sensitivity and spesifity were $77.8 \%$ and $92 \%$ respectively. The difference was statistically significant $(\mathrm{p}<0.001$, Table 2 ). HBME- 1 showed a predominantly membranous pattern in the lesions (Figure $3)$. Forty two cases (42/45 (93.3\%) showed posistive reaction for HBME-1 in FVPC group and 19 of 50 cases (38\%) in FA group. The sensitivity and spesifity were $93.33 \%$ and $62 \%$ between the groups. The difference was also statistically siginificant $(p<0.001$, Table $2)$. Galectin- 3 was seen in 36 cases $((80 \%)$ in FVPC and 23 cases $(46 \%)$ in FA groups. Diffuse cytoplasmic and e few nuclear staining were seen in the neoplastic cells. However vascular structures and inflammatory cells were stained in normal thyroid. The sensitivity and spesifity were $80 \%$ and $54 \%$ between the groups. The difference was statistically significant $(\mathrm{p}<0.001$, Table 2$)$.

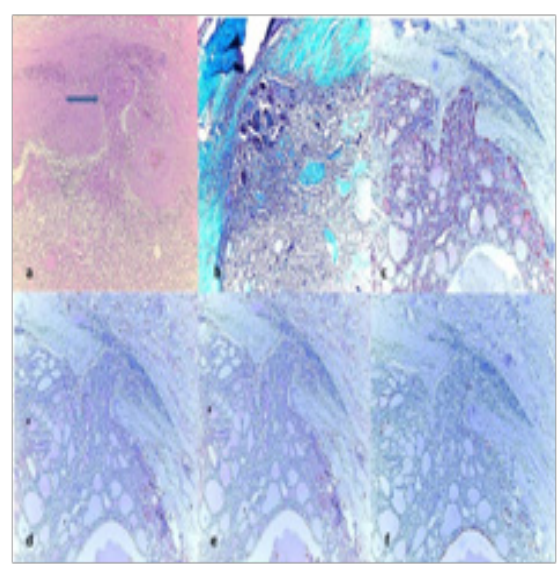

\section{Figure I}

(A) Encapsulated minimally invasive folicular carcinoma (MIFC), showing capsule invasion (arrow) (H\&E, xl00).

(B) Green staining of the capsule with Masson Trichrome (Masson Trichcrome, $x \mid 00)$; The immunohistochemical expressions in tumor.

(C) $(++)$ positive staining of tumor for HBME-I (HBME-I, $x \mid 00)$.

(D) $(++)$ positive staining of tumor for CK-19 (CK-19, xI00).

(E) $(+)$ positive staining of tumor for BRAF (BRAF, $x \mid 00)$.

(F) $(+)$ positive staining of tumor for GAL-3 (GAL-3, $x \mid 00)$.

\section{BRAF, CK-I9, HBME-I, GAL-3 expresssions in FVPC vs MIFC}

BRAF expression was positive in 32 cases $(71.1 \%)$ in FVPC and 16 subjects $(40 \%)$ in MIFC. It was also higher in FVPC than MIFC groups. There was a significant relation between FVPC and BRAF expresssion ( $\mathrm{p}<0.001$, Table 2 ). BRAF staining was heterogenous in MIFC group and was weaker than FVPC. The sensitivity was $40 \%$, the spesifity was $71.11 \%$ among the lesions. According to this data BRAF is more sensitive and spesific in FVPC (Table 2). CK-19 were stained 13 cases $(32.5 \%)$ in MIFC and 35 cases in FVPC (77.8\%). The spesivity was $32.5 \%$ and spesifity was $77 \%$ between the lesions. The difference was significant $(\mathrm{p}<0.001)$. In this connection the following was seen, 36 cases (90\%) were positive in MIFC and 42 cases (93.3\%) were positive in FVPC groups for HBME-1. The differenece was also significant $(\mathrm{p}<0,001)$. The sensitivity and spesifity were $90 \%$ and $93.33 \%$ respectively. When we evaluate GAL-3 in the same way, it was seen this antibody was positive in 36 cases $(80 \%)$ in FVPC and 23 cases $(46 \%)$ in MIFC. The difference was statistically siginifcant between the groups $(\mathrm{p}<0,001$, Tables $1 \& 2)$. 


\section{Figure 2}

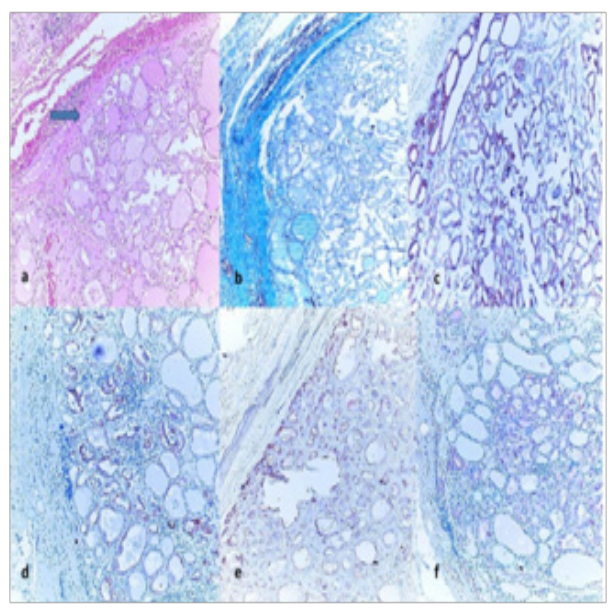

(A) Histology of an encapsulated papillary carcinoma follicular variant (FVPC) showing nuclear transparency $(\mathrm{H} \& \mathrm{E}, \times \mid 00)$

(B) Green staining of the capsule with Masson Trichrome (Masson Trichcrome, $x \mid 00)$; The immunohistochemical expressions in tumor.

(C) $(+++)$ positive staining of tumor for HBME-I (HBME-I, $x \mid 00)$.

(D) $(+++)$ positive staining of tumor for CK-19 (CK-19, $\times 100)$.

$(\mathrm{E})(++)$ positive staining of tumor for BRAF $(\mathrm{BRAF}, \times 100)$.

(F) $(++)$ positive staining of tumor for GAL-3 (GAL-3, xI00).

\section{Figure 3}

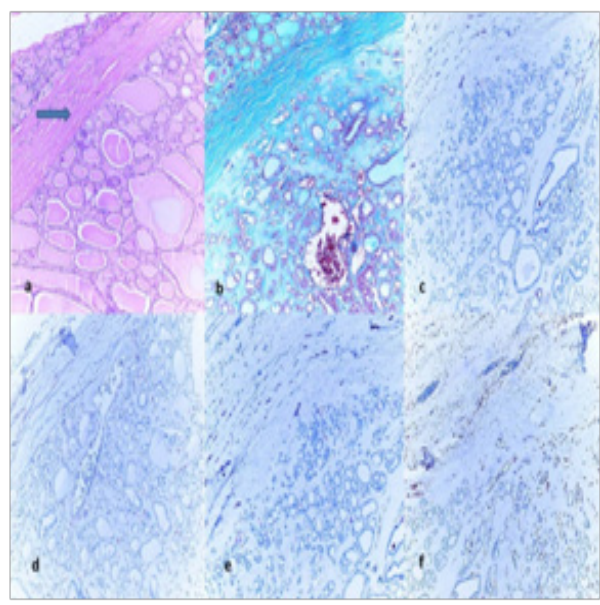

(A) Histology of follicular adenoma (FA) with thick and intact capsule, (H\&E, $\times 100$ ).

(B) Green staining of the capsule with Masson Trichrome (Masson Trichcrome, $x 100$ ); The immunohistochemical expressions in tumor.

(C) $(+)$ negative staining of tumor for HBME-I (HBME-I, $x \mid 00)$.

(D) (+) negative staining of tumor for CK-19 (CK-19, x100).

(E) $(+)$ negative staining of tumor for BRAF (BRAF, $x \mid 00)$.

(F) (+) negative staining of tumor for GAL-3 (GAL-3, xI00).

Table I The effect of immunohistochemical markers for determining the lesion groups

\begin{tabular}{|c|c|c|c|c|c|}
\hline & & \multicolumn{4}{|l|}{ GRUP } \\
\hline & & \multirow{2}{*}{$\begin{array}{l}\text { FA (50) } \\
\text { Count (Row \%) }\end{array}$} & \multirow{2}{*}{$\begin{array}{l}\text { FVPC (45) } \\
\text { Count (Row \%) }\end{array}$} & \multicolumn{2}{|l|}{ MIFC (40) } \\
\hline & & & & Count (Row \% ) & $P$ value \\
\hline \multirow{2}{*}{ Gender } & Female & $38(40,9)$ & $33(35,5)$ & $22(23,7)$ & \multirow{2}{*}{0,074} \\
\hline & Male & $12(28,6)$ & $12(28,6)$ & $18(42,9)$ & \\
\hline \multirow{2}{*}{ CK-19 } & Positive & $4(7,7)$ & $35(67,3)$ & $13(25,0)$ & \multirow{2}{*}{$P<0,00$ I } \\
\hline & Negative & $46(55,4)$ & $10(12,0)$ & $27(32,5)$ & \\
\hline \multirow{2}{*}{ HBME-I } & Positive & $19(19,6)$ & $42(43,3)$ & $36(37,1)$ & \multirow{2}{*}{$P<0,00$ I } \\
\hline & Negative & $3 I(8 I, 6)$ & $3(7,9)$ & $4(10,5)$ & \\
\hline \multirow{2}{*}{ Galectin 3} & Positive & $23(31,1)$ & $36(48,6)$ & $15(20,3)$ & \multirow{2}{*}{$P<0,00$ I } \\
\hline & Negative & $27(44,3)$ & $9(\mid 4,8)$ & $25(4 I, 0)$ & \\
\hline \multirow{2}{*}{ BRAF } & Positive & $14(22,6)$ & $32(5 I, 6)$ & $16(25,8)$ & \multirow{2}{*}{$P<0,00$ I } \\
\hline & Negative & $36(49,3)$ & $13(17,8)$ & $24(32,9)$ & \\
\hline \multirow{2}{*}{ CKI9+HBMEI } & Positive & $19(24,1)$ & $34(43,0)$ & $26(32,9)$ & \multirow{2}{*}{0,001} \\
\hline & Negative & $3 \mid(55,4)$ & II $(19,6)$ & $14(25,0)$ & \\
\hline \multirow{2}{*}{ CKI9+GAL3 } & Positive & $23(31,5)$ & $35(47,9)$ & $15(20,5)$ & \multirow{2}{*}{$P<0,00$ I } \\
\hline & Negative & $27(43,5)$ & $10(16,1)$ & $25(40,3)$ & \\
\hline \multirow{2}{*}{ CKI9+BRAF } & Positive & $14(25,9)$ & $32(5 I, 6)$ & $16(32,9)$ & \multirow{2}{*}{$P<0,00$ I } \\
\hline & Negative & $36(49,3)$ & $13(17,8)$ & $24(25,0)$ & \\
\hline \multirow{2}{*}{ HBMEI+GAL3 } & Positive & $23(26,7)$ & $36(41,9)$ & $27(3 I, 4)$ & \multirow{2}{*}{0,002} \\
\hline & Negative & $27(55, I)$ & $9(18,4)$ & $13(26,5)$ & \\
\hline
\end{tabular}


Table Continued..

\begin{tabular}{|c|c|c|c|c|c|}
\hline & & \multicolumn{4}{|l|}{ GRUP } \\
\hline & & \multirow{2}{*}{$\begin{array}{l}\text { FA (50) } \\
\text { Count (Row \%) }\end{array}$} & \multirow{2}{*}{$\begin{array}{l}\text { FVPC (45) } \\
\text { Count (Row \%) }\end{array}$} & \multicolumn{2}{|l|}{ MIFC (40) } \\
\hline & & & & Count (Row \% ) & $P$ value \\
\hline \multirow{2}{*}{ HBMEI+BRAF } & Positive & $14(18,9)$ & $34(45,9)$ & $26(35, I)$ & \multirow{2}{*}{$P<0,00$ I } \\
\hline & Negative & $36(59,0)$ & $I I(I 8,0)$ & $14(23,0)$ & \\
\hline \multirow{2}{*}{ GAL3+BRAF } & Positive & $\mid 4(2 \mid, 2)$ & $36(54,5)$ & $16(24,2)$ & \multirow{2}{*}{$P<0,001$} \\
\hline & Negative & $36(52,2)$ & $9(13,0)$ & $24(34,8)$ & \\
\hline \multirow{2}{*}{ CKI9+GAL3+HBMEI } & Positive & $19(24,4)$ & $34(43,6)$ & $25(32, I)$ & \multirow{2}{*}{0,001} \\
\hline & Negative & $3 I(54,4)$ & $\operatorname{II}(19,3)$ & $15(26,3)$ & \\
\hline \multirow{2}{*}{ CKI9+HBMEI+BRAF } & Positive & $15(22,4)$ & $27(40,3)$ & $25(37,3)$ & \multirow{2}{*}{0,002} \\
\hline & Negative & $35(5 I, 5)$ & $18(26,5)$ & $15(22,1)$ & \\
\hline \multirow{2}{*}{ HBMEI+GAL3+BRAF } & Positive & $19(28,4)$ & $32(47,8)$ & $16(23,9)$ & \multirow{2}{*}{0,002} \\
\hline & Negative & $3 I(45,6)$ & $13(19,1)$ & $24(35,3)$ & \\
\hline \multirow{2}{*}{$\mathrm{CK} 19+\mathrm{GAL} 3+\mathrm{BRAF}$} & Positive & $15(27,3)$ & $27(49,1)$ & $13(23,6)$ & \multirow{2}{*}{0,005} \\
\hline & Negative & $35(43,8)$ & $18(22,5)$ & $27(33,8)$ & \\
\hline \multirow{2}{*}{$\mathrm{CK} 19+\mathrm{GAL} 3+\mathrm{HBMEI}+\mathrm{BRAF}$} & Positive & $15(20,5)$ & $32(43,8)$ & $26(35,6)$ & \multirow{2}{*}{$P<0,001$} \\
\hline & Negative & $35(56,5)$ & $13(2 \mid, 0)$ & $14(22,6)$ & \\
\hline
\end{tabular}

The pearson chi-square tests show that all the markers assessed single or coexpressed, have statistically significant effect on diagnosing the lesion groups.

Table 2 The effect of immunohistochemical markers for determining the lesion groups

\begin{tabular}{|c|c|c|c|c|}
\hline & & FA & FVPC+MIFC & \\
\hline & & Count (Row \%) & Count (Row \% ) & $P$ value \\
\hline \multirow{2}{*}{ Gender } & Female & $38(40,9)$ & $55(59,1)$ & \multirow{2}{*}{$0,17 \mid$} \\
\hline & Male & $12(28,6)$ & $30(7 I, 4)$ & \\
\hline \multirow{2}{*}{ CK-19 } & Positive & $4(7,7)$ & $48(92,3)$ & \multirow{2}{*}{$P<0,00$ I } \\
\hline & Negative & $46(55,4)$ & $37(44,6)$ & \\
\hline \multirow{2}{*}{ HBME-I } & Positive & $19(19,6)$ & $78(80,4)$ & \multirow{2}{*}{$P<0,00$ I } \\
\hline & Negative & $3 I(8 I, 6)$ & $7(18,4)$ & \\
\hline \multirow{2}{*}{ Galectin 3} & Positive & $23(3 I, I)$ & $5 \mathrm{I}(68,9)$ & \multirow{2}{*}{0,114} \\
\hline & Negative & $27(44,3)$ & $34(55,7)$ & \\
\hline \multirow{2}{*}{ BRAF } & Positive & $14(22,6)$ & $48(77,4)$ & \multirow{2}{*}{0,001} \\
\hline & Negative & $36(49,3)$ & $37(50,7)$ & \\
\hline \multirow{2}{*}{ CKI9+HBMEI } & Positive & $19(24,1)$ & $60(75,9)$ & \multirow{2}{*}{$P<0,00$ I } \\
\hline & Negative & $3 I(55,4)$ & $25(44,6)$ & \\
\hline \multirow{2}{*}{ CKI9+GAL3 } & Positive & $23(31,5)$ & $50(68,5)$ & \multirow{2}{*}{0,149} \\
\hline & Negative & $27(43,5)$ & $35(56,5)$ & \\
\hline \multirow{2}{*}{ CKI9+BRAF } & Positive & $14(25,9)$ & $48(77,4)$ & \multirow{2}{*}{0,001} \\
\hline & Negative & $36(49,3)$ & $37(50,7)$ & \\
\hline \multirow{2}{*}{ HBMEI+GAL3 } & Positive & $23(26,7)$ & $63(73,3)$ & \multirow{2}{*}{0,001} \\
\hline & Negative & $27(55,1)$ & $22(44,9)$ & \\
\hline \multirow{2}{*}{ HBMEI+BRAF } & Positive & $14(18,9)$ & $34(45,9)$ & \multirow{2}{*}{$P<0,00$ I } \\
\hline & Negative & $36(59,0)$ & II $(\mid 8,0)$ & \\
\hline
\end{tabular}


Table Continued.

\begin{tabular}{lllll}
\hline & & FA & FVPC+MIFC & \\
\cline { 3 - 5 } GAL3+BRAF & & Count (Row \%) & Count (Row \% ) & P value \\
\hline \multirow{2}{*}{ CKI9+GAL3+HBMEI } & Positive & $14(21,2)$ & $52(78,8)$ & P<0,00I \\
& Negative & $36(52,2)$ & $33(47,8)$ & \\
CK19+HBMEI+BRAF & Positive & $19(24,4)$ & $59(75,6)$ & P<0,00I \\
& Negative & $31(54,4)$ & $26(45,6)$ & \\
HBMEI+GAL3+BRAF & Positive & $15(22,4)$ & $52(77,6)$ & 0,002 \\
& Negative & $35(51,5)$ & $33(48,5)$ & \\
CK19+GAL3+BRAF & Positive & $19(28,4)$ & $48(71,6)$ & 0,038 \\
& Negative & $31(45,6)$ & $37(54,4)$ & \\
CK19+GAL3+HBMEI+BRAF & Positive & $15(27,3)$ & $40(72,7)$ & 0,049 \\
& Negative & $35(43,8)$ & $45(56,3)$ & \\
& Positive & $15(20,5)$ & $58(79,5)$ & P<0,00I \\
& Negative & $35(56,5)$ & $27(43,5)$ & \\
\hline
\end{tabular}

The pearson chi-square tests show that all the markers assessed single or coexpressed, have statistically significant effect on diagnosing the malignant and benign lesion groups.

\section{BRAF, CK-I9, HBME-I, GAL-3 expresssions in FA vs MIFC}

BRAF expression was positive in 16 cases $(40 \%)$ in MIFC and 14 subjects $(28 \%)$ in FA. It was also higher in MIFC cases. BRAF staining was weak in MIFC. The sensitivity and spesifity were $40 \%$ and $72 \%$, and the difference was significant $(\mathrm{p}<0.001)$. CK-19 were stained 13 cases $(32.5 \%)$ in MIFC and cases in FA (8\%). The spesivity was $32.5 \%$ and spesifity was $92 \%$. The difference was also significant $(p<0.001)$. For HBME-1, 36 cases $(90 \%)$ and 19 cases $(38 \%)$ were stained. The differenece was also significant $(p<0,001)$ and the sensitivity and spesifity were $90 \%$ and $62 \%$. GAL-3 expression was in 15 cases $(37,5 \%)$ in MIFC and 23 cases $(46 \%)$ in FA groups (Table $1)$. The sensitivity and spesifity were $80 \%$ and $54 \%$. The difference was statistically significant $(\mathrm{p}<0.001)$ (Table 2$)$.

Combinations of BRAF , CK-19, HBME-I, GAL-3 immunoreactivity in lesion groups (Table 3) (Table 4)

Binary combinations: In MIFC versus FA groups (Group1), when binary combinations were made with BRAF and the other antibodies with HBME-1, CK-19 or GAL-3 expression, the sensitivity values were $65 \%, 32.5 \%, 37.5 \%$ respectively. For the spesifity, the values were same as $72 \%$. In FVPC versus FA (Group 2), the sensitivity values were close together as $71.11 \%, 60 \%, 71.11 \%$ and for spesifity, the values were the same as $72 \%$. When we considered in a set of the same order to the groups of FVPC and FC (Group 3), the percentages were $65 \%, 32.5 \%, 37.5 \%$ in sensitivity and $71.11 \%, 60 \%, 71.11 \%$ in spesifity. The presentation of the results was same with Group 2 and Group 3 in terms of spesifity. When we considered the malignant lesions (FC and FVPC) and benign lesions (FA) (Group 4) according to the same order of the combinations of these antibodies, the values were $(68.24 \%, 72 \%),(47.06 \%, 72 \%)$ and $(55.29 \%, 72 \%)$ in sensitiviy and spesifity, respectively. In comparing HBME-1 and GAL-3 in group 1, group 2, group 3 and group 4, the sensitivity values were $67.5 \%, 80 \%, 67.5 \%, 74.12 \%$ and spesifity values were $54 \%$ in groups 1,2 and 4 , and $80 \%$ in group 3. The most sensitiviy was in FVPC vs FA. The other combination of HBME-1 with CK-19 showed the sensitivity values as $65 \%, 75.56 \%, 70.27 \%, 75.59 \%$ in groups $1,2,3,4$ respectively. The sensitivity values were the same as $62 \%$ in respect of spesifity in groups $1,2,4$ and $75.56 \%$, in group 3 . The combination of the GAL-3 and CK-19, the percentages were, $37.5 \%$, in group 1 and $3,77.78 \%$ in group 2 and 58.82 in group 4 .

Triple combinations: When we combined the triple combinations of the antibodies in the groups (group 1,2,3,4) with CK-19, HBME-1 and GAL-3, the sensitivity and spesifity rates were as; $62.5 \%, 75.56 \%$, $62.5 \%, 69.41 \%$ in sensitivity and $62 \%, 62 \%, 75.56 \%, 62 \%$ in spesifity in the group $1,2,3,4$, respectively. The most sensitivity was shown in FVPC vs FA as $75.56 \%$ and same rate of spesifity in FVPC vs FC as $75.56 \%$. The rate of sensitivity and spesifity of the combines of CK-19, HBME- 1 and BRAF, the results were as $62,5 \%, 60 \%$, $62.5 \%, 61.18 \%$ and $70 \%, 70 \%, 60 \%, 70 \%$. The results of the other triple combination of the antibodies for HBME-1, GAL-3 and BRAF were $40 \%, 71.11 \%, 40 \%, 56.47 \%$ and $62 \%, 62 \%, 71.11 \%, 62 \%$. The last triple combination was for CK-19, GAL-3 and BRAF and the results were as $32.5 \%, 60 \%, 32.5 \%, 47.06 \%$ and $70 \%, 70 \%, 60 \%$, $70 \%$, respectively.

Four combinations: However the combining of all antibodies for BRAF, CK-19, HBME-1 and GAL-3 showed the sensitivity as $65 \%$, $60 \%, 65 \%, 68.24 \%$ and spesifity as $80 \%, 80 \%, 60 \%, 80 \%$ in groups $1,2,3,4$, respectively.

The specificity and sensitivity of different single and combination of antibodies were calculated. For a single antibody, HBME-1 was the most sensitive for the diagnosis of FVPC vs FA $(93,33 \%)$ whereas CK-19 had the lowest sensitivity among the 4 markers. In contrast, CK-19 has the highest specificity values (92\%) for the diagnosis of all groups. The coexpressions of two marker combinations showed acceptable performances for FVPC vs FA (sensitivity; 60-80\%) and average performances for MIFC vs FA (specificity 54-72\%). HBME$1+$ GAL-3 has the most sensitivity value $(67,5 \%)$ for MIFC vs FA (sensitivity $(80 \%)$ whereas HBME-1+BRAF and GAL-3+BRAF coexpressions have the highest specificity $(72 \%)$ for FC vs FA. Considering both sensitivity and specificity performances, HBME1+BRAF combination was suggested for optimum diagnostic performance. The coexpressions of three antibody combinations 
showed average sensitivity and specificity performances for comparison of the FVPC lesions. The combination of CK-19+GAL3+HBME-1 and CK19+HBME1+BRAF have the highest sensitivity values $(62,5 \%)$ for diagnosing MIFC vs FA. CK-19+GAL-3+HBME-1 has the highest sensitivity value $(75,56 \%)$ for FVPC vs FA. For specificity values, the combination of CK-19+HBME-1+BRAF has the higest specificity value (70\%) for diagnosing MIFC vs FA. The combination of CK-19+GAL-3+HBME1 has the highest specificity value $(75,56 \%)$ for diagnosing MIFC vs FVPC. The coexpression of the four markers showed average sensitivity (60-65\%) and high specificity (80) in group $1,2,3$. For optimum sensitivity and specificity results, the single usage of HBME-1 marker was suggested for the diagnosis of FVPC vs MIFC (sensitivity: 90\%; spesifity: 93,33\%); the single usage of CK-19 marker was suggested for the diagnosis of FVPC vs FA (sensitivity:77,78\%; spesifity:92); and the usage of the combination of the four markers was suggested for the diagnosis of FC vs FA (sensitivity: $65 \%$; spesifity: $80 \%$ ). Immunohistochemical analysis for antibodies was shown in Figures 1-3 for lesion types.

Table 3 CK-19, HBME-I, BRAF and GAL-3 expressions with respect to sensitivity and spesifity among lesion types

\begin{tabular}{|c|c|c|c|c|c|c|c|c|c|c|}
\hline \multirow{2}{*}{ Antibody } & \multirow[t]{2}{*}{$\begin{array}{l}\text { MIFC } \\
=40 n \\
(\%)\end{array}$} & \multirow{2}{*}{$\begin{array}{l}\text { FVPC } \\
=45 n \\
(\%)\end{array}$} & \multirow{2}{*}{$\begin{array}{l}\text { FA = } \\
50 \mathrm{n} \\
(\%)\end{array}$} & \multicolumn{2}{|c|}{$\begin{array}{l}\text { MIFC vs FA } \\
\text { (Group I) }\end{array}$} & \multicolumn{2}{|l|}{$\begin{array}{l}\text { FVPC vs FA } \\
\text { (Group 2) }\end{array}$} & \multirow{2}{*}{$\begin{array}{l}\text { MIFC vs } \\
\text { FVPC } \\
\text { (Group 3) } \\
\begin{array}{l}\text { Sensitivity } \\
(\%)\end{array}\end{array}$} & \multicolumn{2}{|c|}{$\begin{array}{l}\text { (MIFC+FVPC) vs FA } \\
\text { (Group 4) }\end{array}$} \\
\hline & & & & $\begin{array}{l}\text { Sensitivity } \\
\text { (\%) }\end{array}$ & $\begin{array}{l}\text { Specificity } \\
\text { (\%) }\end{array}$ & $\begin{array}{l}\text { Sensitivity } \\
\text { (\%) }\end{array}$ & $\begin{array}{l}\text { Specificity } \\
\text { (\%) }\end{array}$ & & $\begin{array}{l}\text { Sensitivity } \\
\text { (\%) }\end{array}$ & $\begin{array}{l}\text { Specificity } \\
\text { (\%) }\end{array}$ \\
\hline HBME-I & $36(90)$ & $42(93,3)$ & $19(38)$ & 90 & 62 & 93,33 & 62 & 90 & 91,76 & 62 \\
\hline CK-19 & $13(32,5)$ & $35(77,8)$ & $4(8)$ & 32,5 & 92 & 77,78 & 92 & 32,5 & 56,47 & 92 \\
\hline GAL-3 & $15(37,5)$ & $36(80)$ & $23(46)$ & 37,5 & 54 & 80 & 54 & 37,5 & 60 & 54 \\
\hline BRAF & $16(40)$ & $32(7 I, I)$ & $14(28)$ & 40 & 72 & 71,11 & 72 & 71,11 & 56,47 & 72 \\
\hline CKI9+HBME-I & $26(65)$ & $34(75,6)$ & $19(38)$ & 65 & 62 & 75,56 & 62 & 70,27 & 75,59 & 62 \\
\hline CKI9+GAL-3 & $15(37,5)$ & $35(77,8)$ & $23(46)$ & 37,5 & 54 & 77,78 & 54 & 37,5 & 58,82 & 54 \\
\hline CK-19+BRAF & $16(40)$ & $27(60)$ & $14(28)$ & 40 & 72 & 60 & 72 & 40 & 51 & 72 \\
\hline HBME-I+GAL3 & $27(67,5)$ & $36(80)$ & $23(46)$ & 67,5 & 54 & 80 & 54 & 67,5 & 74,12 & 54 \\
\hline HBME-I+BRAF & $26(65)$ & $32(7 I, I)$ & $14(28)$ & 65 & 72 & 71,11 & 72 & 65 & 68,24 & 72 \\
\hline GAL-3+BRAF & $16(40)$ & $32(7 I, I)$ & $14(28)$ & 40 & 72 & 71,11 & 72 & 40 & 56,47 & 72 \\
\hline $\begin{array}{l}\text { CK-19+GAL- } \\
\text { 3+HBME-I }\end{array}$ & $25(62,5)$ & $34(75,6)$ & $19(38)$ & 62,5 & 62 & 75,56 & 62 & 62,5 & 69,41 & 62 \\
\hline $\begin{array}{l}\text { CK-19+HBME- } \\
\text { I+BRAF }\end{array}$ & $25(62,5)$ & $27(60)$ & $15(30)$ & 62,5 & 70 & 60 & 70 & 62,5 & 61,18 & 70 \\
\hline $\begin{array}{l}\text { HBME-I+GAL- } \\
\text { 3+BRAF }\end{array}$ & $16(40)$ & $32(71,1)$ & $19(38)$ & 40 & 62 & 71,11 & 62 & 40 & 56,47 & 62 \\
\hline $\begin{array}{l}\text { CK-19+GAL- } \\
\text { 3+BRAF }\end{array}$ & $13(32,5)$ & $27(60)$ & $15(30)$ & 32,5 & 70 & 60 & 70 & 32,5 & 47,06 & 70 \\
\hline $\begin{array}{l}\text { CK-19+GAL- } \\
\text { 3+HBME- } \\
\text { I+BRAF }\end{array}$ & $26(65)$ & $32(7 I)$ & $15(30)$ & 65 & 80 & 60 & 80 & 65 & 68,24 & 80 \\
\hline
\end{tabular}

Data are given as $\mathrm{n}(\%)$.

Table 4 Table abbreviations.

\begin{tabular}{ll}
\hline BRAF & B-type raf kinase \\
CK-19 & cytokeratin-19 \\
HBME-I & hector battifora mesothelial-I \\
GAL-3 & galectin-3 \\
FA & follicular adenoma \\
MIFC & encapsulated minimally invasive follicular carcinoma \\
FVPC & encapsulated follicular variant of papillary carcinoma \\
PC & papillary carcinoma \\
\hline
\end{tabular}




\section{Discussion}

In this study, we evaluated the immunohistochemical value of BRAF, HBME-1, CK-19, and GAL-3 and their combinations in differential diagnosis of encapsulated follicular patterned thyroid tumors. We found that these markers were higher in malignant follicular lesions (MIFC and FVPC groups) than FA. The follicular lesions presents a heterogeneous group of cases that are neither benign nor atypical for a diagnosis of follicular neoplasm or suspicious for malignancy. The Bethesda 2007 Thyroid Cytology Classification defines these lesions as atypia of undetermined significance/ follicular lesion of undetermined significance. ${ }^{18}$ Furthermore, the National Cancer Institute divided follicular patterned lesions into two diagnostic categories; atypia of undetermined significance/follicular lesion of undetermined significance and suspicious for follicular neoplasm/follicular neoplasm. ${ }^{19}$ The most common problem in thyroid pathology is to differentiate the follicular lesions such as FA, FC and FVPC. ${ }^{3}$

Correct and early diagnosis is necessary to differentiate malign thyroid lesions from bening ones for treatment planning. ${ }^{20}$ No conventional imaging method reliably distinguishes between benign and malignant thyroid nodules. ${ }^{34}$ However, differential diagnosis of thyroid tumors with follicular architecture often presents problems. Papillary and follicular patterns sometimes can be seen in the same lesion in both benign and malignant neoplasms. ${ }^{20}$ Despite welldescribed histopathological criteria, the limited agreement between pathologists evaluating thyroid material is well-documented. ${ }^{21,22}$ Although improved diagnostic techniques allow identification of PC more frequently than in the past, ${ }^{23}$ differential diagnosis of thyroid lesions with follicular pattern is still difficult not only in cytologic material, but also histopathologically. ${ }^{17,24,25}$

Identification of genetic and molecular pathogenesis of malignant thyroid lesions, including activation of some oncogenes, such as BRAF, have provided great insights into tumor development and differential diagnosis of malign lesions. ${ }^{5}$ Many studies focused on gene expression profile of thyroid tumors and its practical role in early differential diagnosis of thyroid lesions. ${ }^{5,26}$ Immunohistochemical markers have commonly been studied as diagnostic tool for differentiation of PC. For example, BRAF mutation is seen commonly in PC cases. Park et al. ${ }^{4}$ reported BRAF mutation in $65.6 \%$ of 278 papillary thyroid micro carcinoma patients and in $67.2 \%$ of 1150 PC patients. In this report, single expressions of BRAF, CK-19 and HBME-1 were significantly higher in malignant lesions whereas GAL-3 was not. The presence of BRAF mutation in $\mathrm{PC}$ is associated with poor clinical prognosis and metastasis. ${ }^{7,27,28}$ In addition to BRAF, protein expression of other genes, such as GAL-3, HBME1, CK-19, were found to be upregulated in PC compared to normal thyroid tissue. ${ }^{26}$ Additionally coexpression of multiple proteins was shown to further increase specificity in differential diagnosis of carcinomas and adenomas. ${ }^{26}$ Nasr et al. ${ }^{29}$ also suggested that the coexpression of HBME-1 and CK-19 has the greater diagnostic utility in the diagnosis of PC than expression of HBME-1 or CK-19 alone. Prasad et al. ${ }^{26}$ studied the 85 carcinomas, 21 adenomas, and 102 non-neoplastic thyroid tissues by immunohistochemistry, and found that expresssion of GAL-3, HBME-1, CK-19, fibronectin-1, and CITED1 was significantly associated with malignancy. Furthermore, co expression of multiple proteins was seen in $95 \%$ of carcinomas and only $5 \%$ of adenomas $(\mathrm{p}<0.0001)$.

In contrary to the vast information on diagnostic potential of immunohistochemical markers for PC, the role and data of the markers to differentiate follicular pattern thyroid lesions are limited.
In the FVPC, BRAF mutation was reported in only $5-20 \%$ of cases. ${ }^{8}$ Adeniran et al. ${ }^{1}$ suggested that BRAF mutation testing can be used with high specificity to predict malignancy in thyroid follicular lesions of undetermined significance. Similarly, we showed that BRAF expression was significantly higher in our FVPC tissues.

In recent studies, BRAF and HBME-1 have been suggested as markers for FVPC of thyroid, and the presence of coexpresssion of BRAF and HBME-1 may increase the specificity of diagnosis. ${ }^{4}$ In our study considering both sensitivity and specificity performances, HBME1+BRAF marker combination was suggested for optimum diagnostic performance. HBME-1 and CITED-1 have been used with increasing frequency to differentiate FVPC from other follicular lesions of thyroid..$^{30}$ However, in the present study we found that HBME-1 expresssion show significant difference between benign and malignant follicular lesion groups. On the other hand, when we analyse the data of all samples, HBME-1 expression in combinations as was significantly higher in BRAF-positive cases. Thus we speculate that HBME-1 expression may show potential for malignance, which can be proven with higher samples sizes. In the literature, othere markers such as CK-19 and GAL-3 exhibit lower sensitivity and specificity in the differential diagnosis of follicular lesions. ${ }^{8}$ In our study, while CK-19 expression was higher in malign follicular lesions (FC and FVPC groups) than FA, with combination only GAL-3 expression was limited in difference between these lesion types.

On the basis of previous reports, ${ }^{31}$ we hypothetized that coexpression of more than one marker would increase diagnostic value, and evaluating the relation between the markers can vary in different lesion groups. HBME-1 and other markers to propose best combination of markers to be used in differential diagnosis of follicular thyroid lesions. Our results showed that only GAL-3 had not significantly higher expression for differantiating malignant and benign lesion groups. Although Lloyd et al.. ${ }^{8}$ Suggested that GAL-3 exhibit lower sensitivity and specificity in the differential diagnosis of follicular lesions, we suggest that diagnostic specificity of GAL-3 may increase when it is combined with CK-19, BRAF and HBME-1. Further studies are needed to evaluate the diagnostic advantages of coexpression of BRAF and GAL-3 in follicular lesions.

The main limitation of the present study is the low number of tissue samples in each follicular diagnostic group. Due to the limited sample size, the immunohistochemical technical variation and evaluation differences of the pathologists could limit the correlations between BRAF, CK-19, HBME-1 and GAL-3 expressions of the follicular patterned thyroid tumors. Definitive conclusions of the study findings can be done. Nevertheless, this is a pilot study on the diagnostic value of immunohistochemical markers in differential diagnosis of follicular lesions of thyroid, and can form a basis for further large scale studies. ${ }^{32}$

\section{Conclusion}

In conclusion, immunohistochemical evaluation of these markers in tyhroid tissue would aid the differentiation of malignant follicular lesions from benign follicular lesions which needs to be confirmed with new antibodies or molecular agents for further studies.

\section{Acknowledgements}

None.

\section{Conflict of interest}

The author declares no conflict of interest. 


\section{References}

1. Adeniran AJ, Hui P, Chhieng DC, et al. BRAF mutation testing of thyroid fine-needle aspiration specimens enhances the predictability of malignancy in thyroid follicular lesions of undetermined significance. Acta Cytol. 2011;55(6):570-575

2. Ito Y, Yoshida H, Tomoda C, et al. HBME-1 expression in follicular tumor of the thyroid: an investigation of whether it can be used as a marker to diagnose follicular carcinoma. Anticancer Res. 2009;25(1A):179-182.

3. LiVolsi VA, Baloch ZW. Follicular neoplasms of the thyroid: view, biases, and experiences. Adv Anat Pathol. 2004;11(6):279-287.

4. Xu X, Quiros RM, Gattuso P, et al. High prevalence of BRAF gene mutation in papillary thyroid carcinomas and thyroid tumor cell lines. Cancer Res. 2003;63(15):4561-4567.

5. Eze OP, Starker LF, Carling T. The role of epigenetic alterations in papillary thyroid carcinogenesis. J Thyroid Res. 2011. 7 p.

6. Park YJ, Kim YA, Lee YJ, et al. Papillary microcarcinoma in comparison with larger papillary thyroid carcinoma in BRAF(V600E) mutation, clinicopathological features, and immunohistochemical findings. Head Neck. 2010;32(1):38-45.

7. Prasad ML, Pellegata NS, Huang Y, et al. Galectin-3, fibronectin-1, CITED-1, HBME1 and cytokeratin-19 immunohistochemistry is useful for the differential diagnosis of thyroid tumors. Mod Pathol. 2005;18(1):4857.

8. Mase T, Funahashi H, Koshikawa T, et al. HBME-1 immunostaining in thyroid tumors especially in follicular neoplasm. Endocr $J$. 2003;50(2):173-177.

9. Sahoo S, Hoda SA, Rosai J, et al. Cytokeratin 19 immunoreactivity in the diagnosis of papillary thyroid carcinoma: a note of caution. Am J Clin Pathol. 2001;116(5):696-702.

10. Aron M, Kapila K, Verma K. Utility of galectin 3 expression in thyroid aspirates as a diagnostic marker in differentiating benign from malignant thyroid neoplasms. Indian J Pathol Microbiol. 2006;49(3):376-380.

11. Mehrotra P, Okpokam A, Bouhaidar R, et al. Galectin-3 does not reliably distinguish benign from malignant thyroid neoplasms. Histopathology. 2004;45(5):493-500.

12. Nasr MR, Mukhopadhyay S, Zhang S, et al. Immunohistochemical markers in diagnosis of papillary thyroid carcinoma: Utility of HBME1 combined with CK19 immunostaining. Mod Pathol. 2006;19(12):16311637.

13. Beesley MF, Mc Laren KM. Cytokeratin 19 and galectin-3 immunohistochemistry in the differential diagnosis of solitary thyroid nodules. Histopathology. 2002;41(3):236-243.

14. Schlumberger MJ. Papillary and follicular thyroid carcinoma. $N$ Engl J Med. 1998;338(5):297-306.

15. Joo JY, Park JY, Yoon YH, et al. Prediction of occult central lymph node metastasis in papillary thyroid carcinoma by preoperative BRAF analysis using fine-needle aspiration biopsy: a prospective study. J Clin Endocrinol Metab. 2012;97(11):3996-4003.
16. Mataraci EA, Ozgüven BY, Kabukçuoglu F. Expression of cytokeratin 19, HBME-1 and galectin-3 in neoplastic and nonneoplastic thyroid lesions. Pol J Pathol. 2012;63(1):58-64.

17. Scognamiglio T, Hyjek E, Kao J, et al. Diagnostic usefulness of HBME1, galectin-3, CK19, and CITED1 and evaluation of their expression in encapsulated lesions with questionable features of papillary thyroid carcinoma. Am J Clin Pathol. 2006;126(5):700-708.

18. Cibas ES, Ali SZ. The bethesda system for reporting thyroid cytopathology. Thyroid. 2009;9(11):1159-1165.

19. Faquin WC, Baloch ZW. Fine-needle aspiration of follicular patterned lesions of the thyroid: Diagnosis, management, and follow-up according to national cancer institute (NCI) recommendations. Diagn Cytopathol. 2010;38(10):731-739

20. Cooper DS, Doherty GM, Haugen BR, et al. Management guidelines for patients with thyroid nodules and differentiated thyroid cancer. Thyroid. 2006;16(2):109-142.

21. Guerra A, Fugazzola L, Marotta V, et al. A high percentage of BRAFV600E alleles in papillary thyroid carcinoma predicts a poorer outcome. J Clin Endocrinol Metab. 2012;97(7):2333-2340.

22. Hofman V, Lassalle S, Bonnetaud C, et al. Thyroid tumours of uncertain malignant potential: frequency and diagnostic reproducibility. Virchows Arch. 2009;455(1):21-33.

23. Davies L, Welch HG. Increasing incidence of thyroid cancer in the United States, 1973-2002. JAMA. 2006;295(18):2164-2167.

24. Lloyd RV, Buehler D, Khanafshar E. Papillary thyroid carcinoma variants. Head Neck Pathol. 2011;5(1):51-56.

25. Xing M, Westra WH, Tufano RP, et al. BRAF mutation predicts a poore clinical prognosis for papillary thyroid cancer. J Clin Endocrinol Metab. 2005;90(12):6373-6379.

26. Saggiorato E, Cappia S, De Giuli P, et al. Galectin-3 as a presurgical immunocytodiagnostic marker of minimally invasive follicular thyroid carcinoma. J Clin Endocrinol Metab. 2001;86(11):5152-5158.

27. Hirokawa M, Carney JA, Goellner JR, et al. Observer variation of encapsulated follicular lesions of the thyroid gland. Am J Surg Pathol. 2002;26(11):1508-1514

28. B Zhang, Jiang YX, Liu JB. Utility of contrast-enhanced ultrasound for evaluation of thyroid nodules. Thyroid. 2010;20(1):51-57.

29. Nga ME, Lim GS, Soh CH, et al. HBME-1 and CK19 are highly discriminatory in the cytological diagnosis of papillary thyroid carcinoma. Diagn Cytopathol. 2008;36(8):550-556.

30. Wallander M, Layfield LJ, Jarboe E, et al. Follicular variant of papillary carcinoma: reproducibility of histologic diagnosis and utility of HBME1 immunohistochemistry and BRAF mutational analysis as diagnostic adjuncts. Appl. Immunohistochem. Mol Morphol. 2010;18(3):231-235.

31. Nikiforov YE. RET/PTC rearrangement in thyroid tumors. Endocr Pathol. 2002;13(1):3-16.

32. Utiger RD. The multiplicity of thyroid nodules and carcinomas. $N$ Engl J Med. 2005;352(23):2376-2378. 